\title{
Multisensor systems for gas analysis: optimization of arrays for classification of pharmaceutical products
}

\author{
I.V. Kruglenko, B.A. Snopok, Yu.M. Shirshov, F.J. Rowell* \\ V. Lashkaryov Institute of Semiconductor Physics, National Academy of Sciences of Ukraine \\ 41, prospect Nauky, 03028 Kyiv, Ukraine \\ *Centre for Pharmaceutical and Environmental Analysis, School of Science, University of Sunderland, UK
}

\begin{abstract}
In the present work, the use of the cluster analysis method in the "fuzzy logic" concept for the optimization of the cross-selective sensor arrays ("electronic nose", EN) is considered. This approach enables to purposefully form the sensor arrays with definite chemical functionality optimized for the solution of the specific applied problems. The criteria of the optimization of the sensor response, number and type of the sensor elements are considered with the goal to improve the classification of widely used pharmaceutical products. The optimization of EN array in the kinetic mode and selection of the most informative part of the sensor response enabled to reduce the analysis time, and also the number of sensors in array, to improve the discriminatory capability of the whole array. Being based on the analysis of response kinetic peculiarities, the physical mechanisms determining the peculiarities of adsorption-desorption processes at the interface have been considered.
\end{abstract}

Keywords: electronic nose, identification, multisensor system, cluster analysis, fuzzy logic.

Paper received 17.03.04; accepted for publication 17.06.04.

\section{Introduction}

Development and investigation of the chemical and biochemical sensors as a separate direction on the boundary of physics, chemistry and biology is intensively developed in recent years [1-3]. The important place among such systems is occupied by selective sensors aimed at the single components of gas mixtures - sensors of oxygen, hydrogen as well as other constituents of gas mixtures; these systems use the concept of specific sensors built on the principal "one gas - one sensor". Despite wide spectra of available sensors, they can't provide the respective description of the multicomponent mixtures that are characteristic for the chemical environment of the natural origin $[4,5]$. Besides, the creation of highly selective coatings sensitive only to the specific molecules, is practically impossible, because the compounds presenting the same chemical class have functional groups of the similar spatial structure with the close physical and chemical properties.

This put forward as a task of paramaunt importance to create multisensor systems for the analysis of the composition of the multicomponent chemical media (MCM), which can be used for the ecological monitoring, control of the technological processes, analysis of the products quality and for the medical and biological investigations [6-9]. Systems of that type are intensively developed in many countries, with the aim of their use for the control of the media, which are vitally important for human beings. Thus, the medicine and pharmacy are one of the largest markets for such systems, which require the development of the new intelectual diagnostics. Indeed, even B.C. the possibility of using the odors for the diagnostics of different deseases was known. Thus, the development of the intellectual gas matrixes and following development of the "electronic nose" must provide more wide use of the "scent" diagnostics in medicine. At the same time, high effeciency (and, thus, a respective danger in the case of the unproper use) of the modern pharmaceutical products supposes the necessity of the quality control of the products and also their identification in the case of the need, because they can cause latent and evident nonwanted or adverse effects for patients. Among the teratogenic, that is such products that can cause adverse effects, are such medicine (drugs) as alkaloids, quinine, tetracycline, sulfanilamides, etc. Thus, the express identification of the widely used pharmaceutical products is practically very important.

The "Electronic nose" is designed, first of all, for the identification of the various MCM. The necessary condition for this is the ability of the multisensor array to classify MCM: the increase of the discriminating ability of the array is of primary importance. But the ways of the optimization of the sensor array towards respective necessary sensitivity of the sensitive layers and ways of the parametrization of the sensor response still remain with- 


\section{I.V. Kruglenko et al.: Multisensor systems for gas analysis: optimization of the array for ...}

out answer. Besides, the problem of the "superfluous" sensors with the small information capacity is very important due to "additional information noise" of such sensors [9]. Indeed, in connection with the complex nature of the interaction between MCM and sensor elements, data on them have multidimensional and diverse character, so before analysis it is unclear, how essential is this or another property for identification of the certain analyte or class of analytes. At given conditions, in the first place is the problem to classify the multidimensional data; that is the necessity to optimize grouping from the viewpoint of the best correspondence between obtained results and the final target of this classification. In contrast to many statistical procedures, the methods of the cluster analysis enable to determine the "most probable solution", when the identification problem is still in the descriptive stage of investigation. This enables to develop the approach concerning the ways of the optimization of the sensor array and to give it certain chemical functionality.

The objective of the present work was to consider ways of optimization of the multisensor arrays for the gas analysis with the aim to improve the quality of pharmaceutical product classification by using the methods of the cluster analysis that are based on the concept of the "fuzzy logic".

\section{Multisensor systems of gas analysis: a general approach}

The approach to the analysis of the multicomponent chemical mixtures of the unknown composition can be borrowed from the analysis of the principles, which are habitual to the life support systems of the biological organisms, in particular, olfaction. Olfaction system of the animals and human beings is the unique instrument for the analysis of the chemical components in air [11-13]. This system is characterized by: (i) high rapidity of the analysis (milliseconds); (ii) wide sensitivity range (up 9 orders in concentration); (iii) low detection limits; (iv) memory possibility, possibility to distinguish complex mixtures of compounds and (v) to identify fragrant compounds in the complex mixtures; (vi) miniature sizes.

The idea of MCM detection consists in the simultaneous analysis of signals from the system of sensitive elements, each element has unique selectivity profile. That is, MCM can be described not by the sum of its constituent components, but some model with the characteristic for each MCM set of parameters - by a chemical image (CI) $[14,15]$. Instrumental system for the formation of CI with the consequent identification using the methods of pattern recognition are called as "electronic nose", EN $[16,17]$. The basis of EN is the array of sensors with the sensitive layers possessing different chemical functionality. Such systems creates "image" in terms of the sensitive element signal values in the form of a multidimensional surface of responses. The approach enables to get over the principal limitations of the specific sensors and provide unlimited quantity of the possible "images" of different components. Between the probe image in the "chemical" base (concentration of the separate components on axes) and its analog in the response space of the sensor array one can observe the one-to-one correspondence (Fig. 1). Because the dimensionality of the response space is sufficiently smaller than the dimensionality of the "chemical basis", the sensors must be weakly selective and have a wide dynamical range of their signal change. In this case, the commonly used sensitivity level to the wide spectrum of the chemical compounds will be provided, and the necessary number of the different combinations in the response space will be achieved $[18,19]$.

The first intellectual model of EN, which included elements of pattern recognition, was developed in 1982 by Persaud and Dodd [20]. After that the different vesions of EN, which were based on various physical converters, were developed, which enabled to obtain simple, compact, completely automated devices suitable to solve several applied problems $[6,7,16,17]$. But, as a whole, the question concerning formation of the necessary for the specific application, of the chemical functionallity of the sensor array was open, due to, at first place, problem of the provision of the uniqueness for the each separate image of MCM of different origin.

\section{Piezoelectric converters for EN systems}

Among physical converters of various types used in EN devices, piezoelectric converters are among most attractive, because these enable to develop approach, which supposes separation of the chemical interaction processes and its physical transformation into the respective sensor response [21]. The dependence of the resonant frequency $f$ of an acoustic oscillator on the mass of substance $\Delta m$ that is on its surface is used in a Quartz Crystal Microbalance (QCM) to register intermolecular interactions. The linear dependence between the resonant frequency shift $\mathrm{f}$ of QCM and $\mathrm{m}$, which is supposed in many cases [22], in reality is idealized representation obtained in the conditions that the density and viscosity of the sensitive layer do not differ from material of the converter itself and do not change during its interaction with analyte. But in such cases, when the hydrodynamic interaction between the physical converter and environment can be neglected (mainly in measurements in a gas phase), and to consider a sensitive layer as solid, then the mass change $\Delta m$ with the change of the oscillation frequency $\Delta f$ of the piezocrystal resonator should be described by the expression [23]:

$$
\Delta m=S \cdot \rho_{Q} \cdot d_{Q}\left(-\frac{\Delta f}{f_{0}}+\frac{1}{f_{0}} \cdot \frac{d f}{d P} \cdot P+\frac{1}{f_{0}} \cdot \frac{d f}{d T} \cdot T\right)
$$

where $S$ is the electrode area; $\rho_{Q}$ and $d_{Q}$-density and thickness of the quartz plate with the surface layer; $f_{0}$ natural oscillation frequency of the piezoelement. If it is possible to neglect temperature and pressure changes during the experiment $(d f l d P=0, d f l d T=0)$, then the equation (1) is transformed into the well-known Sauebrey equation [24]: 


\section{I.V. Kruglenko et al.: Multisensor systems for gas analysis: optimization of the array for ...}

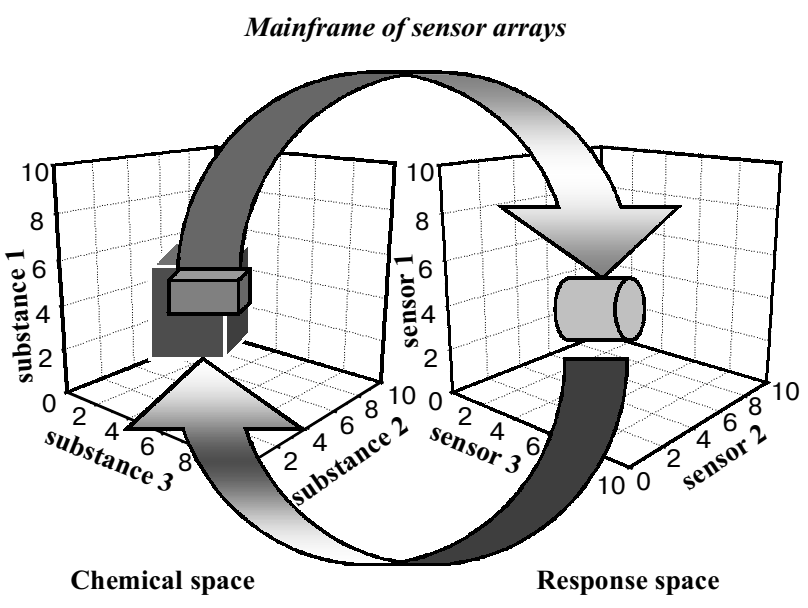

Fig.1. General functioning procedure of the multisensor arrays.

$$
\Delta f=-C_{Q} \cdot \Delta m / S
$$

where $C_{Q}$ is the integral resonator sensitivity to the additional mass on the surface. That is, the crystal oscillation frequency is linearly changed with the mass change on its surface. For the AT-cut of the quartz crystal with main frequency $10 \mathrm{MHz}$ and $0.25 \mathrm{~cm}^{2}$ area, the converter sensitivity is about $1 \mathrm{ng} \cdot \mathrm{Hz}^{-1}$. Such ratio is valid only for the small mass changes $\Delta m$ on the converters surface, which is determined by sorption of the analyte by the sensitive layer. This mass depends on the gas volume $V$, which flows over the surface of the piezocrystal and can be written as:

$$
V=q \cdot t=\Delta M / C \quad \text { or } \quad \Delta M=C \cdot q \cdot t
$$

where $C$ is the analyte concentration in the gas flow, $\Delta M-$ analyte mass in the gas flow, $q$ - gas flow velocity, and $t$ - time. If $\gamma$ is the effective coefficient of analyte capture by the sensitive layer $(\Delta m=\gamma \cdot \Delta M)$, then in the region far from saturation, the converter frequency shift will depend on gas flow parameters in the following way (equation (2) and (3)):

$\Delta f=\left(C_{Q} / S\right) \cdot(\gamma \cdot C \cdot q \cdot t)$

Thus, the frequency shift of the crystal oscillations depends on time, analyte concentration and carrier gas velocity. If the analysis time will be fixed under the constant velocity of the gas flow, then the change of the crystal frequency is directly proportional to the analyte concentration in the gas flow. It is evident that the various processes can lead to the violation of the conditions in which the equation (4) was obtained. Among them - adsorption of the mixture on the walls, fluctuations of the temperature, pressure, carrier gas velocity, etc. The standartization of the analysis conditions, choice of the respective materials, and the increase of the wall temperature are widely used methods to solve these problems. Besides, in these cases when the flowing measurements regime is used, the contribution of these factors is negligible.

\section{Sensitive layers for EN}

Piezocrystalline detector is the powerfull analytic device; this is clear from the proportion for the frequency change to the analyte concentration (about $1 \mathrm{ng} \cdot \mathrm{Hz}^{-1}$ ). Taking into account that the piezosensor is non-specific device, the detector reveals the frequency change due to mass deposition of any substance onto the surface. Thus, for the formation of the selectivity profile of the sensitive element, it is necessary to choose the coatings that will be sensitive to the respective analytes. In this case, the procedures of the formation of sensitive architectures with the receptor centers on the surface of the physical converters have a paramount importance because of the necessity of the molecula recognition principles realization in the case, when receptor centers of various origin and structure are fixed in the two-dimensional matrix at the phase interface (homogenic-heterogenic processes).

Some information concerning the ways to solve this problem is provided by odor systems developed on the base of analysis of human beeings and animals scent peculiarities. For example, in accordance with the Amoore system, the "form" of the molecule determines its odor [25]. This stereochemical concept has seven "primary" odors, for each of them the sizes of the receptor "holes" were calculated. Thus, the receptor centers that provide several contacts of different nature with the respective accomodation in the space of the pair receptor-analyte, are capable to provide the necessary profile of the selective layer, in general. Taking into account the ability of the organic materials concerning formation of various types of intermolecular interactions and their diversity, exactly the organic compounds are most attractive for the formation of the sensor arrays of a new generation. Additional information concerning the use of organic materials in sensor technique can be found in [26-28].

\section{Formation and recognition of $\mathrm{CI}$}

The above considered ways of the creation of intellectual sensor systems assume the presence of interaction of MCM target components with sensitive layers, the peculiarities of which unambigously characterize the given type of the product. But the response of the sensors also depends on the procedure of the probe preparation and analysis of the samples prehistory, irregularity of the gas flow, etc., can lead to the unpredicted shape of the kinetics curve at the initial stage [10, 29]. Besides, the part of the signal that corresponds to the stationary level, also doesn't have additional information about MCM [30]. Indeed, the increase of the identification capability of the "electronic nose" in the dynamic regime was shown using the linear classificator - the principal component analysis. Neuron networks (non-linear classificator) confirmed this result. As was supposed, for the different classificators the best results on the quality of recognition are achieved when the initial part and stationary state are excluded from the input data, that is the kinetics information enables to provide the required level of the 


\section{I.V. Kruglenko et al.: Multisensor systems for gas analysis: optimization of the array for ...}

uniqueness of the selectivity profile as a single sensor element as well as of the chemical image as a whole [2931]. At the same time, the developed approaches don't enable their effective use as an instrument to optimize the sensor array: absence of the simple and understandable classification standard and rigid requirements towards quantity of the necessary set of data essentially limit possibilities of these methods as a useful experimental tools in the given case. Let us consider the possible ways for the solution of this problem.

Classification means here division of some set of objects or observations into uniform, in certain sense, groups - clusters, elements of which are similar, at the same time between clusters the qualitative differences are observed [32]. Thus, the objective of the cluster analysis is the separation of the internal structure, in general case multidimensional data, and attributing each of them from the given set of objects to one of the class (sort). The cluster analysis can be considered as one of the methods of data compression, when to the big quantity of images (for example, observations, which differ as a consequence of the uncontrolled analysis conditions) is made corresponded to the limited number of clusters, using some function of mutual correspondence. The classical methods of the cluster analysis lead to separation of the data set into clusters with clearly determined boundaries; this means that irrespectively to the quality of the input data they must be related to the certain class. In fact, this means that the part of the data will be classified incorrectly, this is of course impermissible for a number of branches such as medical diagnostics or the life support control at big industrial enterprises with the continuous production cycle. In order to prevent such inaccurate classification, the methods based on the concept of "fuzzy logics" were developed, where the concept of diffuse or fuzzy set is used. In contrast to the classification according to the prototype, "indistinctness" arises in the situation when attributing to the certain category is assumed true only to a certain degree. The source of such "typicality" can be graduation concepts (a priori information about classes - separation methods) or possibility to distinguish "more" or "less" succesfull solutions by the analysis of the algorithm peculiarities of the standard or function of the class identity (hierarchy approaches). It is necessary to note that just the separation methods are a powerful instrument, when the task is in the optimization of the acquirement procedure optimization of the multidimensional data set with the aim of their following use in the procedures of the image recognition.

The most important element of any optimization procedure is the availability of the classification degree; which enables to evaluate expediency of the use of different sensors in the array and enables to separate the most informative part of the multidimensional response surface. The variant of cluster analysis in the Rousseeuw version enables to obtain such estimation, by using the so-called $s(i)$ (silhouette width) as a parameter [33, 34]. The minimization of the respective target function for the certain combination of the variables and observations enables to calculate $s(i)$ both for a single observation and for its average value $S(i)$ in the whole data set. Because $s(i)$ is in fact the characteristic of the attribution to the certain cluster, then the $s(i)$ close to unity means that data in the cluster are compactly placed, especially if all $s(i)$ values for all the cluster elements have comparable values. Small $s(i)$ values testify that the given observation lies between regions of the compact clusters, - that is remaining within the concept of the "fuzzy logic" it is impossible to make a conclusion to which cluster the given measurement belongs. Thus, despite lowering the classification ability of the methods based on the given concept (because the quantity of the solutions on the attribution to the certain cluster is decreased), number of the wrong classifications is practically reduced to zero. Negative $s(i)$ value points that the given observation most probably is wrongly attributed to the given cluster - that is it cannot be correctly placed to any of the clusters formed from the considered observation.

Presented short review of the cluster analysis peculiarities based on the concept of "fuzzy" logic enables to propose the method for the optimization of the multisensor arrays for specific application, using the distribution of $s(i)$ as the criterion. Thus, the optimal combination of the sensitive elements and procedures of the parametrization of the sensor response must provide the formation of the uniform clusters in the response space, which are characterized by the high and close $s(i)$ values both for single observations within cluster, and the mean $S(i)$ value for all observations as a whole. We will consider the realization of this approach for the classification of three types of medical compounds.

\section{Experiment}

Conceptually the development of the EN systems supposes the solution of the four main problems, namely: (i) synthesis of the receptor centers with the specified chemical functionality; (ii) their integration with a physical converter; (iii) complete integration of the separate components of the system with taking into account the peculiarities of the probes preparation and carrying out of the measurements; (iv) parametrization of the response of the sensor array, formation of the data bases of standardized chemical images. In this case, the principle question which is applied for all development stages of such systems is the search for the ways of the informativity increase of the separate observation and the efficiency of the conversion of the chemical information into the mathematical model.

\section{Materials}

Calixarenes, tert-bytil-calix[4]aren (C[4]A), tert-bytilcalix[6]aren (C[6]A) and tert-bytil-calix[8]aren (C[8]A) were kindly provided by professor Kalchenko V.I. (Institute of Organic chemistry, NAS Ukraine, Kyiv). Linear polyacenes: pentacen, tetracen, and phthalocyanine $\left(\mathrm{H}_{2} \mathrm{Pc}\right)$ were kindly provided by professor Vertsimakha Ya.I. (Institute of Physics, NAS Ukraine, Kyiv). Annu- 


\section{I.V. Kruglenko et al.: Multisensor systems for gas analysis: optimization of the array for ...}

lenes: dibenzotetraazaannulene ( $\mathrm{H}_{2}$ TAA) and its methyl substituted derivative $\left(\mathrm{H}_{2}\right.$ TMTAA) were kindly provided by professor Lampeka Ya.D. (Institute of physical chemistry, NAS Ukraine, Kyiv). All compounds were used without additional purification. The films of organic compounds were obtained by thermal vacuum evaporation (VUP-5M, pressure $5 \cdot 10^{-4} \mathrm{~Pa}$ ) on the metal electrodes of PEC (from one side). The mean condensation rate was 10 $\mathrm{nm} / \mathrm{min}$; temperature of QCM was $297 \pm 2^{\circ} \mathrm{K}$. The thickness of the films during evaporation process was controlled by the quartz resonator and was equal to $100 \mathrm{~nm}$.

The following three types of the medicines were investigated: Excedrin ${ }^{\circledR}$, Bristol-Myers SQUIBB Co. (USA); Groseptol $480^{\circledR}$, Polfa Grodzisk (PL); Ampicillini trihydras-Darnitsa ${ }^{\circledR}$, Darnitsa (UA). The experimental volume corresponded to the one pill crushed into powder. The gas "image" of the pharmaceutical compounds, where inorganic salts, cellulose and its derivatives are usually used as fillers, is mainly determined by the active substance (usually organic substance with the small volatility), ingredients (such as benzol acid, mineral oils, etc.), and also aromatizators. In the case of the absence of the latter ones and sufficiently low volatility of the active components, the gas "image" of the compound is characterized by the low concentration of the target-oriented substances in the gas phase and by the change of CI with time due to the change of (both qualitative and quantitative) a gas phase composition over the solid probe. Respectively, all measurements were carried out only on the samples dispersed immediatly after unpacking before the analysis.

\section{Experimental setup}

Multisensor QCM analyzer of the gas mixtures contain: (i) temperature controlled measurement camera with the sensor matrix of the flowing type; (ii) quartz generators block; (iii) block of the frequency measurement and RS232 sequential interface constructed on the base of a specialized microprocessor (AT89C2051); (iv) generator of the gas mixtures; $(v)$ system collection and processing of the information on the base of personal computer [32]. The system temperature was maintained at $37 \pm 0.3^{\circ} \mathrm{C}$ level, flowrate of the carier gas (argon) was about $180 \mathrm{ml} / \mathrm{min}$. The measurement procedure included the following stages: gas circulation up to the frequency stabilization of the sensors $( \pm 3 \mathrm{~Hz})$; circulation of the vapor-gas mix-

Table 1. Classification ability of the triple sets for three types of the pharmaceutical products. Data set includes $i V$-parameters for 1.4...5.2 min.; $I C$ and $S(i)$ are presented only for the sets with adequate classification. Sets with partial classification are not shown

\begin{tabular}{|c|c|c|c|c|c|c|c|}
\hline \multicolumn{3}{|c|}{ Sensor name } & Ampicillini $^{\circledR}$ & Excedrin $^{\circledR}$ & Groseptol $480^{\circledR}$ & $I C$ & $S(i)$ \\
\hline tetracene & pentacen & $\mathrm{C}[4] \mathrm{A}$ & + & + & + & 96.61 & 0.59 \\
\hline tetracene & pentacen & $\mathrm{C}[6] \mathrm{A}$ & + & + & + & 96.43 & 0.6 \\
\hline tetracene & $\mathrm{C}[4] \mathrm{A}$ & $\mathrm{C}[8] \mathrm{A}$ & + & + & + & 89.62 & 0.41 \\
\hline tetracene & $\mathrm{C}[4] \mathrm{A}$ & $\mathrm{Pc}$ & + & + & + & 91.38 & 0.53 \\
\hline tetracene & $\mathrm{C}[4] \mathrm{A}$ & $\mathrm{H}_{2}$ TMTAA & + & + & + & 84.45 & 0.48 \\
\hline tetracene & $\mathrm{C}[6] \mathrm{A}$ & $\mathrm{C}[8] \mathrm{A}$ & + & + & + & 91.23 & 0.41 \\
\hline pentacen & $\mathrm{C}[4] \mathrm{A}$ & $\mathrm{C}[6] \mathrm{A}$ & + & + & + & 97.66 & 0.69 \\
\hline pentacen & $\mathrm{C}[4] \mathrm{A}$ & $\mathrm{C}[8] \mathrm{A}$ & + & + & + & 89.23 & 0.56 \\
\hline pentacen & $\mathrm{C}[4] \mathrm{A}$ & $\mathrm{Pc}$ & + & + & + & 96.58 & 0.7 \\
\hline pentacen & $\mathrm{C}[4] \mathrm{A}$ & $\mathrm{H}_{2} \mathrm{TAA}$ & + & + & + & 87.49 & 0.66 \\
\hline pentacen & $\mathrm{C}[4] \mathrm{A}$ & $\mathrm{H}_{2}$ TMTAA & + & + & + & 92.62 & 0.52 \\
\hline pentacen & $\mathrm{C}[6] \mathrm{A}$ & $\mathrm{C}[8] \mathrm{A}$ & + & + & + & 90.37 & 0.55 \\
\hline pentacen & $\mathrm{C}[6] \mathrm{A}$ & $\mathrm{Pc}$ & + & + & + & 97.01 & 0.73 \\
\hline pentacen & $\mathrm{C}[6] \mathrm{A}$ & $\mathrm{H}_{2}$ TMTAA & + & + & + & 92.98 & 0.44 \\
\hline $\mathrm{C}[4] \mathrm{A}$ & $\mathrm{C}[6] \mathrm{A}$ & $\mathrm{Pc}$ & + & + & + & 97.54 & 0.62 \\
\hline $\mathrm{C}[4] \mathrm{A}$ & $\mathrm{C}[6] \mathrm{A}$ & $\mathrm{H}_{2} \mathrm{TAA}$ & + & + & + & 98.75 & 0.47 \\
\hline $\mathrm{C}[4] \mathrm{A}$ & $\mathrm{C}[6] \mathrm{A}$ & $\mathrm{H}_{2} \mathrm{TMTAA}$ & + & + & + & 97.63 & 0.48 \\
\hline $\mathrm{C}[4] \mathrm{A}$ & $\mathrm{Pc}$ & $\mathrm{H}_{2} \mathrm{TAA}$ & + & + & + & 87.06 & 0.55 \\
\hline $\mathrm{C}[4] \mathrm{A}$ & $\mathrm{Pc}$ & $\mathrm{H}_{2}$ TMTAA & + & + & + & 96.69 & 0.47 \\
\hline $\mathrm{C}[6] \mathrm{A}$ & $\mathrm{Pc}$ & $\mathrm{H}_{2} \mathrm{TAA}$ & + & + & + & 90.52 & 0.61 \\
\hline $\mathrm{C}[6] \mathrm{A}$ & $\mathrm{Pc}$ & $\mathrm{H}_{2}$ TMTAA & + & + & + & 96.42 & 0.41 \\
\hline $\mathrm{C}[4] \mathrm{A}$ & $\mathrm{H}_{2} \mathrm{TAA}$ & H2TMTAA & + & + & + & 81.82 & 0.43 \\
\hline . $\ldots \ldots \ldots \ldots \ldots$ & $\ldots$ & & & & & & \\
\hline $\mathrm{C}[6] \mathrm{A}$ & $\mathrm{C}[8] \mathrm{A}$ & $\mathrm{H}_{2} \mathrm{TMTAA}$ & - & - & - & - & - \\
\hline $\mathrm{C}[8] \mathrm{A}$ & $\mathrm{Pc}$ & $\mathrm{H}_{2} \mathrm{TAA}$ & - & - & - & - & - \\
\hline $\mathrm{C}[8] \mathrm{A}$ & $\mathrm{Pc}$ & $\mathrm{H}_{2} \mathrm{TMTAA}$ & - & - & - & - & - \\
\hline $\mathrm{Pc}$ & $\mathrm{H}_{2} \mathrm{TAA}$ & $\mathrm{H}_{2}$ TMTAA & - & - & - & - & - \\
\hline
\end{tabular}




\section{I.V. Kruglenko et al.: Multisensor systems for gas analysis: optimization of the array for ...}

ture; purging by carrier gas up to the restoration of the initial frequency value of the QCM. The scheme of the measurement part of the device is presented in Fig. 2.

\section{Data processing}

The responses of the sensors based on the piezoelectric converters can be characterized by the value of the response frequency (in the different moments of time), its change rate, integral characteristics and their combinations. Respectively, the formation of CI was carried out using three parameters: frequency change rate of the sensors $\Lambda$, saturation level $\Delta f_{s}$ and $i V$-parameter. The $\Lambda$ parameter was calculated at the initial part of the kinetic curve and $\Delta f_{s}$ was determined by the averaging of the experimental values after reaching the stationary state of the response; $i V$-parameter was calculated at different times $t^{t h}$ by numerical integrating of the experimental dependencies with the following division of the value of integral on $t^{t h}$ values [17, 31]:

$$
i V\left(t_{1}, t_{2}\right)=\frac{1}{t_{1}-t_{1}} \int_{t_{1}}^{t_{2}} \Delta f(t) d t .
$$

Taking into account, that CI should not depend on absolute values of the sensor responses, CI probes were normalized by unity (that is the sum of the responses for all sensors for each analysis was equal to unity). To process of the experimental data, the programs Origin (MicroCal. Software, Inc) were used. Statistical analysis was carried out using S-PLUS 2000 (Math. Soft, Inc).

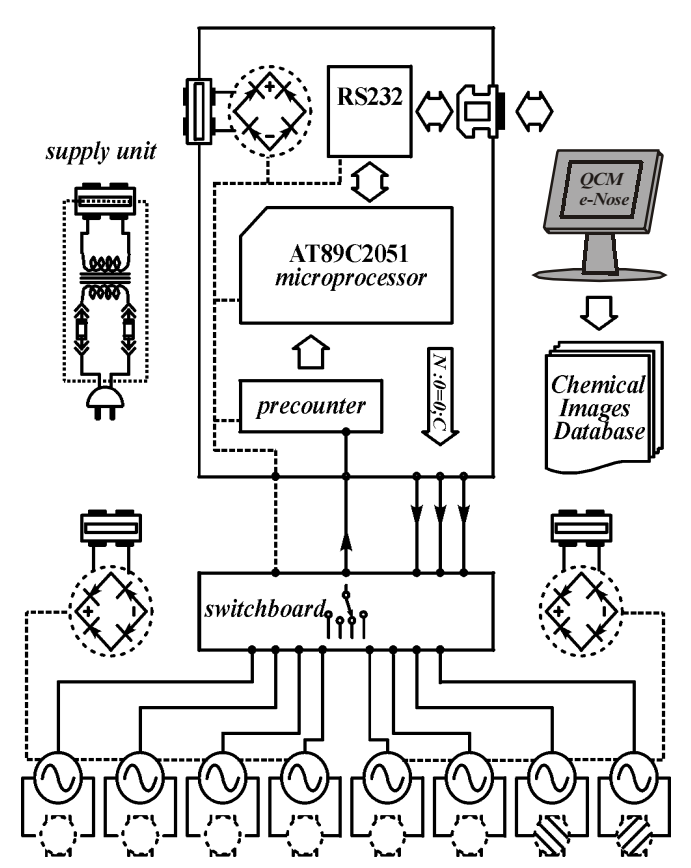

Fig. 2. Scheme of the measurement part of the experimental setup.

\section{Results}

Analysis of the sensor responses for these three types of pharmaceutical compounds (Fig. 3) enabled to attract attention to the following peculiarities in the kinetic behaviour of the curves: $(i)$ for all the samples, the curves are monotonic; (ii) the saturation levels are similar for some sensors; (iii) response of the sensors respectively to their values is differently changed with time for different sensors; (iv) transition to the equilibrium state is achieved after various times for different sensors. The qualitative comparison of the sensor response with the aim to find more stable and specific data parametrization for CI formation, with the use of the signal growth rate at the initial part $\Lambda$ and the saturation level $\Delta f_{s}$ for $\mathrm{CM}$ formation didn't enable to obtain the unique CI like to many other cases [29-31]. As the use of these parameters didn't enable even to qualitatively identify these three samples, the $i V$-parameter was calculated, because its use have positive effect on the degree of the identification for different types of sensors in many cases.

As $i V$-parameter can be calculated for different time intervals, initially it was necessary to determine the most informative part of the kinetic curve, using the degree of the clusterization as the criterion. Data matrix for cluster analysis included $i V$-parameters for three samples for the each type of drugs; variants of the paramerization were considered for time intervals: $1 \ldots 2 ; 1.3 \ldots 2.6$; $1.4 \ldots 5.2 ; 1.1 \ldots 6.8 ; 5 \ldots 7 \mathrm{~min}$. In Fig. 4 the dependencies of the average value $S(i)$ for the different time intervals are presented. As can be seen from the figure at time discretion from 1.4 to $5.2 \mathrm{~min}$., the highest $S(i)$ values are observed. Moreover, in this case single $s(i)$ values within classes have approximately equal values (Fig. 5). It is interesting that the use of the wide interval of the input data (1.1..6.8 min.) provide lower classification ability in comparison with the best interval in the region of the strongest signal change of sensors (Figs 3 and 5). Thus, for the following analysis the interval time between 1.4 and $5.2 \mathrm{~min}$ of the sensor response was chosen.

The analysis of the kinetic curves in Fig. 3 shows that some curves have similar character of relaxations (both for the different sensors for the same sample and for one sensor but three various samples). This testifies that not only optimal time but also quantity and type of the sensors can essentially influence the classification task. That is why, it is important to ascertain how the discriminatory ability depends on the type of the sensor coating. Taking into account the statistical character of the task, it is relatively hard to establish the tendency of the influence at a large number of variables (sensors). Therefore, the number of sensors in the subset was reduced up to three. It is understandable that the reduction of the number of sensors effectively decreases the number of the possible combinations in the response space. But from the practical point of view, even for three sensors with the respective selectivity profile and 10 grades of the signal, the number of the possible combinations exceeds $10^{4}$ (above $3^{10}$ ). At the same time, for three pharmaceutical 

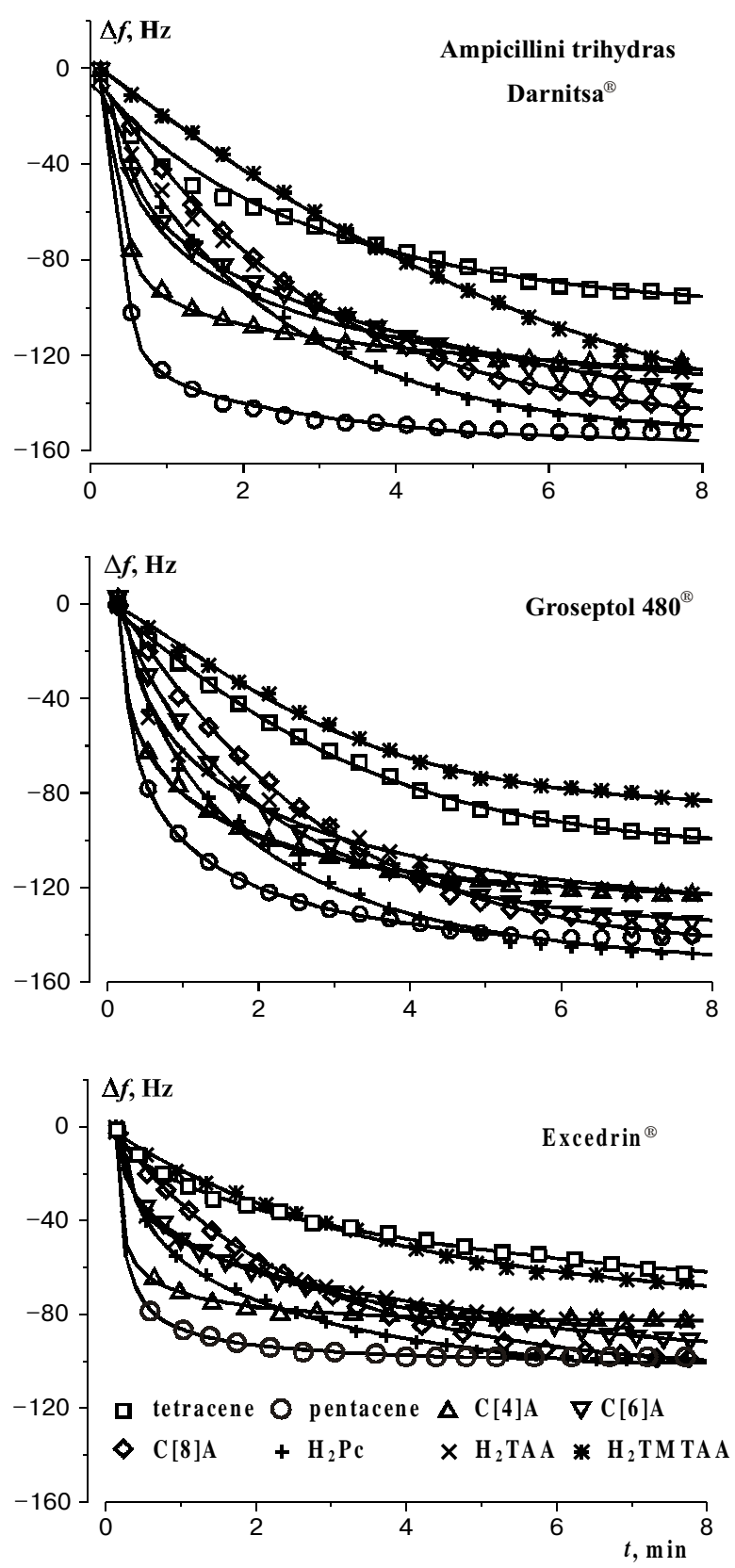

Fig. 3. Experimental dependencies of the frequency on time for three pharmaceutical products.

compounds under consideration, the decrease of the number of sensors from 8 up to 3 optimal results in the growth of $S(i)$ from 0.49 up to 0.73 (Table 1); and uniformity $s(i)$ inside clusters is increased - the clusters become more compact (Fig. 5).

As is known, the cluster analysis can't enable at once to find the optimal set of the sensors. So, the ability to classify was estimated using various combinations of the sensors (YES or NO for the right classification, see Table 1). Besides, for the different combinations of three sensors (in all 54 sets) the discriminatory ability was estimated by $S(i)$ value in the case when the classification was adequate (all YES). Thus, the analysis of the classification results for the different subsets provided possibility to evaluate the influence of the sensors on the dis-

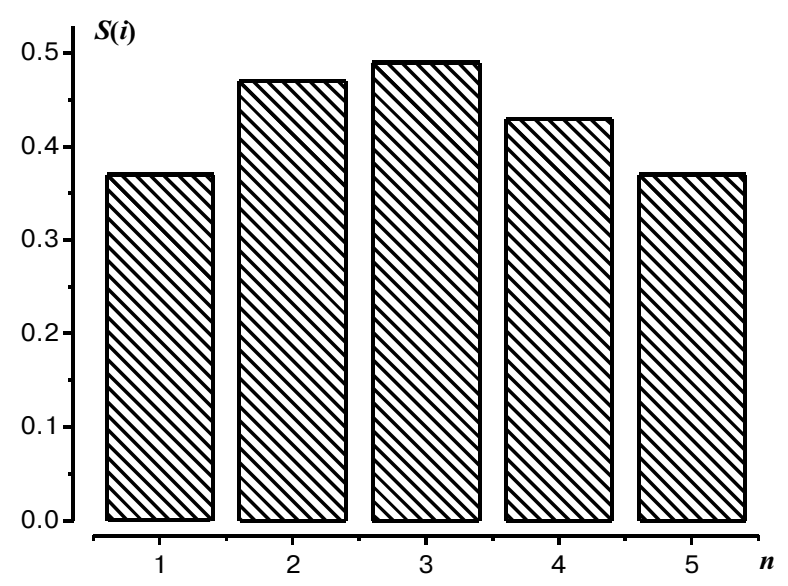

Fig. 4. Dependence of $S(i)$ on $i V$-parameter for different time intervals: $1 \ldots 2(1) ; 1.3 \ldots 2.6(2) ; 1.4 \ldots 5.2$ (3); $1.1 \ldots 6.8$ (4); $5 \ldots 7$ (5) min. For the cases (1) and (5) $S(i)$ value is presented for the comparison because the classification wasn't adequate.

criminatory capability (Table 1). The highest discriminatory ability was obtained for the set C[6]A, pentacene, $\mathrm{H}_{2}$ Pc. It is interesting to compare the statistics of the entry of the various sensors into triple subsets with the right classification. From these 22 combinations C[4]A (13), C[6]A (11), pentacene (10), $\mathrm{H}_{2} \mathrm{Pc}$ (9), $\mathrm{H}_{2}$ TMTAA (7), tetracen (6), C[8]A and $\mathrm{H}_{2}$ TAA (4). At the same time, for the triple sets with $S(i)>0.6$ (6 combinations) we have: C[4]A (4), C[6]A (4), $\mathrm{H}_{2} \mathrm{Pc}$ (4), pentacene (4) and $\mathrm{H}_{2}$ TAA (2): for three best $(\mathrm{S}(\mathrm{i}) \geq 0.69)$ - pentacene (3), $\mathrm{H}_{2} \mathrm{Pc}(2)$, C[6]A (2) and C[4]A (2). It should be emphasized that for the latter three ones the two-dimensional representation preserves about $97 \%$ of information of the initial data sets (IC, see Table 1). Thus, from the eight sensitive layers, the sensors which are based on pentacene, C[4]A, C[6]A, $\mathrm{H}_{2} \mathrm{Pc}$ and $\mathrm{H}_{2}$ TAA coatings are essential for the high discriminatory capability, when tetracene, $\mathrm{C}[8] \mathrm{A}$ and $\mathrm{H}_{2}$ TMTAA have small informational capacity.

It is interesting to note that the different from the chemical point of view compounds were included into the best sets. In respect to this, the question arises if it is possible to establish the connection of the peculiarities of the molecular structure of these compounds, their behaviour under their interaction with the analytes of different origin with their contribution to the discriminatory capability of the multisensor array?

\section{Discussion}

The considered above testifies that it is the kinetic information that enables to obtain the most unique CI for various MCM; the presence of the experimental curves enables to compare them with the theoretical dependencies within the frames of the certain model of adsorption with the aim to clarify the mechanism of the process which proceeds at the interface.

The approximation of the kinetic dependencies, presented in Fig. 3 by different analytical functions has shown that all curves are adequately described by the function of the following type: 
I.V. Kruglenko et al.: Multisensor systems for gas analysis: optimization of the array for ...
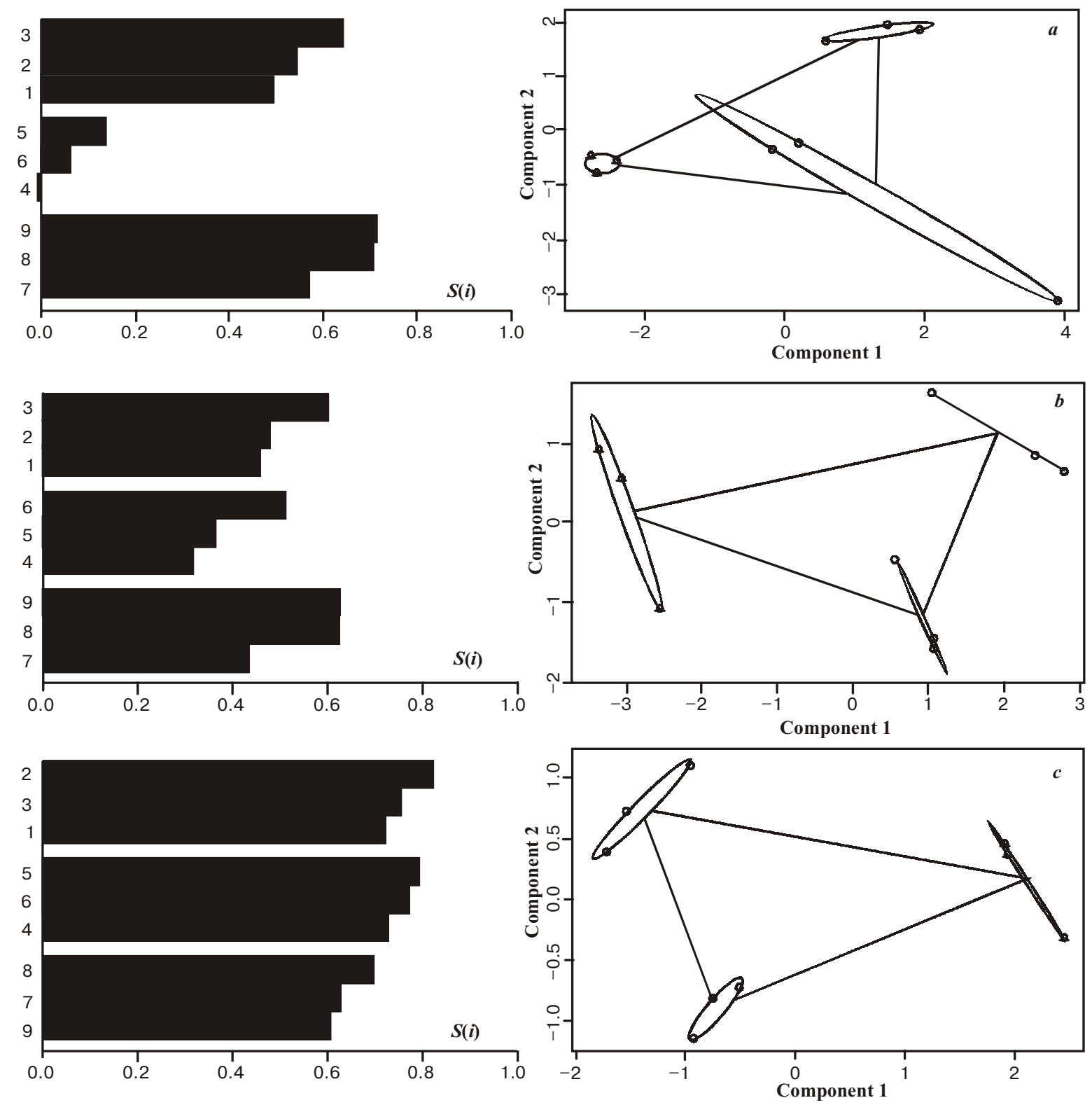

Fig. 5. The values of $s(i)$ (left) and and classification of clusters (right) for 8 sensors ( $i V$-parameter for 1.1...6.8 min.) $-a$; for 8 sensors $\left(i V\right.$-parameter for 1.4..5.2 min.) - $b$ : for 3 sensors (C[6]A, pentacene, $\mathrm{H}_{2} \mathrm{Pc}$; iV-parameter for 1.4 ...5.2 min.) - c. Ampicillini trihydras-Darnitsa ${ }^{\circledR}(1,2,3)$, Excedrin ${ }^{\circledR}(4,5,6)$, Groseptol $480^{\circledR},(7,8,9)$.

$$
\Delta f(t)=\Delta f_{s} \cdot\left(1-\exp \left(-(t / \tau)^{\beta}\right)\right)
$$

where $\Delta f_{s}$ - limiting value, $\tau$ - characteristic time, and $\beta$-parameter, the value of which is within the $[0,1]$ interval. The average values for the parameters of the equation (6) for the considered samples are presented in Table 2 . At $\beta=1$, the equation (6) transforms into the classical Langmuir dependence. It is interesting, that at $t \rightarrow 0$ (6) transforms into the known kinetic Bangham-Bart equation [35]:

$$
\Delta f(t)=A \cdot t^{\beta}-B
$$

where $A, B$ and $\beta$-constants, which depend on the nature of the adsorbate-adsorbenpaart. The Bangham kinetics is usually connected to the either non-uniformity of the adsorbent surface or to the interaction in the adlayers.
The concept of the non-uniform surface, as well as concept of the interaction are two basic classical approaches, which enable to explain the deviations from the Langmuir theory [36]. The reasons of such deviations are understandable: not only concentration limitations are distorting the statements of the Langmuir model, but the real surface itself is essentially different from the ideal - it is the spatially organized systems in nano-micro scale. The peculiarities of the organization depend on the molecular structure, fabrication procedure, used substrate material, etc. The concept of the heterogeneous surface supposes that the surface is characterized by some distribution of the heats of adsorption. This can be a result of the presence of adsorption centers of different types (in relation to the molecules of the specified type) and the 


\section{I.V. Kruglenko et al.: Multisensor systems for gas analysis: optimization of the array for ...}

fluctuations of the adsorption heat as a consequence of the influence, for example, of the surface structure, as well. Thus, the peculiarities of the real surface, can be taken into account by the respective choice of the distribution type of the differential heat of adsorption. Taking into account the relation between differential heat of adsorption and the rate constant $\tau$ in the equation (6) [36], it is possible to formulate the statement also in kinetic terms: the respective choice of the distribution of the relaxation times is the characteristic feature of the given surface.

The formulation of the problem in the terms of relaxation times enables to reveal the physical meaning of the equation (6). Taking into account its coincidence with the widely known KWW equation (KohlrauschWilliams-Watts, KWW, "stretched exponential" function), it is reasonable to suppose and general physical mechanism which determines the origin of such dependencies [37-39]. It is known that KWW enables to adequately describe the wide spectrum of physical phenomena in various materials [40-42], the characteristic feature of which is the presence of the set of relaxation periods in the system. This law isn't the empirical observation and has theoretical background [38, 43]: the relaxation of the system in the conditions of the stochastic fluctuations has the form $\sim \exp \left(-(t / \tau)^{\beta}\right)$. This conclusion doesn't depend on the microscopic physical model of the process, and is determined explicitly by the stochastic nature of the relaxation process. It is interesting to note that the analysis within the frames of the percolation theory shows a direct link between KWW type of relaxation and the fractal nature of material [44]. For example, the presence of the spatial fractal structures must result in the appearance of KWW types of relaxation in them, and vice versa, if the system is characterized by KWW relaxation dependencies, this is indicative of the fractal properties of the object itself.

The analysis of the parameters of the equation (6) calculated and averaged for all the samples (Table 2) shows the presence of the correlation between them: for $\mathrm{C}[8] \mathrm{A}$, $\mathrm{H}_{2}$ TMTAA and tetracene $\tau$ are minimal and $\beta$ close to 1.0 (Langmuir model, "slow" responce), for C[4]A and pentacene $-\beta$ are maximal at $\beta \leq 0.5$ (that is, the sensor response is probably limited by the mass transfer proc-

Table 2. Average values of the parameters of the equation (6) for three types of pharmaceutical products.

\begin{tabular}{|c|c|c|c|c|}
\hline № & $\begin{array}{l}\text { Sensor } \\
\text { name }\end{array}$ & Average $\beta$ & $\tau, \min$ & \\
\hline 1. & tetracene & $0.90 \pm 0.10$ & $20 \ldots 30$ & |"Slow" adsorption \\
\hline 2. & pentacene & $0.50 \pm 0.01$ & $1 \ldots 10$ & “Quick” adsorption \\
\hline 3. & $\mathrm{C}[4] \mathrm{A}$ & $0.35 \pm 0.01$ & $1 \ldots 10$ & "Quick" adsorption \\
\hline 4. & $\mathrm{C}[6] \mathrm{A}$ & $0.55 \pm 0.05$ & $10 \ldots 20$ & "Mean" adsorption \\
\hline 5. & $\mathrm{C}[8] \mathrm{A}$ & $0.90 \pm 0.10$ & $19 \ldots 21$ & "Slow" adsorption \\
\hline 6. & $\mathrm{H}_{2} \mathrm{Pc}$ & $0.65 \pm 0.10$ & $10 \ldots 17$ & "Mean" adsorption \\
\hline 7. & $\mathrm{H}_{2}$ TAA & $0.60 \pm 0.10$ & $14 \ldots 20$ & "Mean" adsorption \\
\hline 8. & $\mathrm{H}_{2}$ TMTAA & $0.95 \pm 0.05$ & $20 \ldots 50$ & "Slow" adsorption \\
\hline
\end{tabular}

SQO, 7(2), 2004 esses, "quick" response); for $\mathrm{C}[6] \mathrm{A}, \mathrm{H}_{2} \mathrm{Pc}$, and $\mathrm{H}_{2} \mathrm{TAA}-$ $\tau$ average at $0.5<\beta<0.7$. It is necessary to note that obtained values of $\tau$ and $\beta$ are characteristics of the sensitive layer itself, not the analyte. This enable to suggest that $\beta$ parameter can be some characteristic of the sensitive coating essential for the optimization of such crossselective arrays.

These results can be understood within the frame of the phenomenological model of the adsorption-desorption on the surface [45]. Corresponding to this approach, the adsorption-desorption processes can be characterized as those controlled by the energy profile on the surface. One of the main results of this method is the classification of the systems on "quick" or "slow" adsorbents in dependence on the relative values of the surface adsorption and desorption barriers. "Slow" and "quick" systems show very different surface dynamics (Table 2). Indeed, in the case of the strong adsorbents, adsorption time of particle near the surface is much smaller, then during desorption - such systems influence equilibrium surfacevolume and cause the limitation of the mass transfer near/ on the surface. For "slow" adsorbents the desorption time is much smaller then the particle adsorption time (the Langmuir model), that is, the surface structure almost doen't influence the dynamical behaviour of the particle near/on the surface due to its "energetic" inertia. At the same time, for the "quick" adsorbents the structure peculiarities will determine the adsorption-desorption equilibrium on the nanostructured surface due to influence on the surface energy profile. It is understandable, the means of the surface structurization on the micro- and nano- scale in such a manner enable to control the selectivity profile of these systems. The obtained result enables to consider the correlation between chemical functionality of the sensitive layers of the EN systems and their molecular structure. All molecules used in the given work as components of sensitive layers consist of the aromatic fragments with the hydrophilic and hydrophobic regions, which creates adsorption centers for the respective molecules. Besides, the presence of the nanovoids in calixarenes can stimulate additional steric stabilization of the analyte-receptor complex. Thus, the functional possibilities for the diffeent types of the molecular structures are determined in the first place by the dominating (from the viewpoint of ability to form intermolecular complexes) peculiarities of their molecular structure: high polarizability of pentacen and $\mathrm{H}_{2} \mathrm{Pc}$ [46]; the presence of the intramolecular hydrogen bonds in $\mathrm{Pc}$ and $\mathrm{H}_{2}$ TAA [47]; by the presence of the nanovoids in calixarenes [48]. It is necessary to underline that the form of nanocavities of calixarenes in the solid state is changing from the truncated cone $(\mathrm{C}[4] \mathrm{A})$ through cylinder (C[6]A) up to irregular figures for $(\mathrm{C}[8] \mathrm{A})$; this is the result of the enlargement of the intramolecular hydrogen bond [49]. This determines C[8]A structure disorder in the solid state, which distorts the nanovoids and complicates interaction of the possible analyte with the intramolecular polar part. Thus, tetracen, $\mathrm{H}_{2}$ TMTAA and C[8]A (the latter ones due to their spatial structure) have almost hydrophobic surface under absence of the possibilities of the additional 


\section{I.V. Kruglenko et al.: Multisensor systems for gas analysis: optimization of the array for ...}

intermolecular interactions. C[4]A among considered calixarenes most fully maintain its structure in the solid state, and, respectively, the ability to bound complementary analytes due to rigid structure of the nanovoid, which essentially increase the lifetime of the analyte in the adsorbed state. Pentacen, in its turn, having high polarizability, is capable to form Van-der-Waals complexes of different origin with wide spectrum of the various analytes. $\mathrm{H}_{2} \mathrm{Pc}, \mathrm{C}[6] \mathrm{A}$ and $\mathrm{H}_{2}$ TAA, besides that are capable to interact with different analytes, both hydrophobic and hydrophilous, which enables to form multicontact complexes with the different origin of the intermolecular bonds.

\section{Conclusions}

The considered comparisons of the results of cluster analysis and approximation of the kinetic dependencies enables to made a conclusion about the correlation between $\beta$-parameter and the efficiency of the classification task: optimally sensitive are such layers which are characterized by the values of $\beta<<1$ - this provides, on the one hand, the sufficiently wide selectivity profile of the sensitive layer, and, on the other hand, the possibility to control the selectivity profile of the sensitive element by using the surface structurization in the nano-micro-scale.

\section{Acknowledgements}

Authors are thankfull to the international science foundation INTAS for the financial support of this work (projects 00-00870 and 01-00257).

\section{References}

1. J.R. Stetter, W.R. Penrose // Journal of The Electrochemical Society, 150, pp. S11-S16, (2003).

2. R. Brown, E. Zellers, Environmental Monitoring, in SensorsA Comprehensive Survey Grandke and W.H.Ko, eds., VCH Publishers, Weinheim: FRG, (1989)

3. C.L. Honeybourne // Journal of Chemical Education, 77(3), pp. 338-343 (2000)

4. F. Avila, D.E. Myers, Palmer C // J. Chemometrics, 5, pp. 455-465 (1991).

5. K.J. Albert, D.R. Walt, D.S. Gill, T.C. Pearce // Anal., 73, pp. 2501-2508 (2001).

6. J.W. Gardner, P.N. Bartlett // Sensors and actuators B., 18, pp. 211-220 (1994).

7. H.T. Nagle, R.G. Osuna, S.S. Schiffman // IEEE Spectrum, Special issue on Electronic nose, pp. 22-38 (1998).

8. R. Lucklum, P. Hauptmann // Sensors and Actuators B, 70, pp. 30-36 (2000).

9. I.V. Kruglenko, B.A. Snopok // Proceedings of The Ninth International Symposium on Olfaction and Electronic Nose (Ed. Arnaldo D'Amico and C.Di Natale), MMIII Aracne Editrice S.r.1., pp.104-111 (2003)

10. M. Sharaf, D. Illman, B. Kowalski, Chemometrics, J. Willey \& Sons, (1986)

11. T.C. Pearce // BioSystems, 41, pp. 43 (1997).

12. A.K. Vidybida // Sur.Biophys, 29, 7B2 (2000).

13. K. Mori, H. Nagao, Y. Sasaki // Comput. Neural. Syst., 9, P. 79R (1998).

14. W.Goepel // Sensors and Actuators B, 52, pp.125 (1998).
15. F. Davide, A. D'Amico // Sensors and actuators A, 32 , pp. 507-518 (1992)

16. J.W. Gardner, P.N. Bartlett, Electronic Noses. Principles and Applications, Oxford, University Press, (1999).

17. B.A. Snopok, I.V. Kruglenko // Thin Solid Films, 418 (1), pp. 2141 (2002).

18. K. Faber, A. Lorber, B. Kowalski // Jornal of Chemometrics, 11, pp.419 (1997).

19. F.Davide, A. D'Amico // Sensors and Actuators A, 32 pp. 507 (1992)

20. K.C. Persaud, J.Dodds // Nature, 299, pp. 352 (1982).

21. T. Tatsuma, Y. Watanabe, N. Oyama // Anal. Chem., 71, pp. 3632-3636 (1999).

22. W.P. Carey, B.R. Kowalski // Anal. Chem., 58, pp. 3077-3084 (1986).

23. G.B. Altshuler, N.N. Efimov, V.G. Shakulin, Quartz generators., M.: Radio I svyaz', (1984).

24. G. Sauebrey // Z.Phys. 155, pp.206-222 (1959).

25. J.E. Amoore, Stereochemical theory of Olfaction // Nature, 198, pp. 271-272 (1964).

26. F.L. Dickert, R. Sikorski // Materials Science and Engineering, C 10, pp. 39-46 (1999).

27. Zhong Cao1, Kazutaka Murayama, Katsuyuki Aoki // Analytica Chimica Acta., 448, pp.47-59 (2001).

28. Di Natale C., Paolesse R., Macagnano A., Mantini A., MariP., D'Amico A // Sensors and Actuators B, 68, pp.319$323(2000)$.

29. T. Ekloev, P. Martensson, I. Lundstroem // Analytica Chimica Acta, 381, pp. 221-232 (1999).

30. Kruglenko I.V., Snopok B.A., Shirshov Yu.M, Reznik A.M., Nowicki D.W., Dekhtyarenko A.K., Sensors for Environmental Control (Ed.P.Siciliano), World Scientific Publishing Co.Pte.Ltd., pp.239-243 (2003).

31. I.V. Kruglenko, B.A. Snopok, Y.M. Shirshov, E.F. Venger // Semiconductor Physics, Quantum Electronics and Optoelectronics, 3(4), pp. 529-541 (2000).

32. Kaufman, L. and Rousseeuw, P.J. Finding, Groups in Data: An Introduction to Cluster Analysis, Wiley, New York., (1990).

33. P.J. Rousseeuw // J. Comput. Appl. Math., 20, pp. 53-65 (1987).

34. A. Struyf, M. Hubert, and P.J. Rousseeuw, // Computational Statistics and Data Analysis, 26, pp. 17-37 (1997).

35. M. Breusse, L. Faure, B. Claudel, J. Veron // Progress in Vacuum Microbalance Technique, 2, pp. 229 (1973).

36. F.F. Volkenshtein, Electronic processes on the surfaces of semiconductors at hemosorption, M:Nauka. (1987).

37. R. Metzler, J. Klafter // Physics Reports, 339, pp. 1-77 (2000).

38. I. Koponen // Journal of Non-Crystalline Solids, 189, pp. 154160 (1995)

39. K. Weron, A. Jurlewicz // J.Phys.A: Math. Gen., 26, pp. 395410 (1993).

40. B.J. West // Chemical Physics 284, pp. 45-57 (2002).

41. M. M. Ahmada, K. Yamadaa, T. Okudaa // Carbohydrate Polymers, 53, pp. 289-296 (2003)

42. I. Avramov, I. Gutzow // Journal of Non-Crystalline Solids, 298, pp. 67-75 (2002).

43. O. Edholm, C. Blomberg // Chemical Physics, 252, pp. 221225 (2000).

44. I.Avramov, V.Tonchev // Journal of Non-Crystalline Solids, 194, pp. 122-128 (1996).

45. O.V.Bychuk, B.O'Shaughnessy // Journal of Colloid and Interface Science, 167, pp.193-203 (1994).

46. M. Pope, C.E. Swenberg, Electronic Processes in Organic Crystals and Polymers (2nd Edition), Oxford University Press (1999).

47. Andre Zh, Simon Zh.-Zh. Andre, Molecular semiconductors, M.: "Mir" (1988).

48. Richard M. Crooks, Antonio J. Ricco // Accounts of Chemical Research, 31(5), pp. 219-225 (1998).

49. C.D.Gutsche. Calixarenes, Royal Society of Chemistry, Cambridge (1989). 